\title{
An Innovative Eigenvector-Based Method for Traffic Light Scheduling
}

\author{
Mohammad Amin Soltani-Sarvestani, ${ }^{1}$ Zohreh Azimifar ${ }^{1},{ }^{1}$ Alexander Wong, \\ and Ali Akbar Safavi ${ }^{1}$ \\ ${ }^{1}$ School of Electrical and Computer Engineering, Shiraz University, Shiraz, Iran \\ ${ }^{2}$ Department of Systems Design Engineering, University of Waterloo, Waterloo, Canada \\ Correspondence should be addressed to Zohreh Azimifar; azimifar@cse.shirazu.ac.ir
}

Received 6 February 2020; Revised 13 August 2020; Accepted 16 September 2020; Published 30 September 2020

Academic Editor: Giuseppe Guido

Copyright (C) 2020 Mohammad Amin Soltani-Sarvestani et al. This is an open access article distributed under the Creative Commons Attribution License, which permits unrestricted use, distribution, and reproduction in any medium, provided the original work is properly cited.

\begin{abstract}
This paper introduces two traffic light strategies to control traffic and avoid traffic jam in urban networks. One strategy is a new traffic light scheduling system, which controls traffic light using local variables (waiting time and number of vehicle on links) but has a global impact on the traffic, using shared variables between neighbour intersections. The proposed traffic light scheduling system is designed based on eigenvector centrality of intersection relation matrix. The intersection relation matrix is a new representation of a junction which indicates the traffic relation between intersection's links and adjacent intersections. The second contribution is expanding a new dual mode traffic light strategy (namely, Exit Status Traffic Light (ETL)), which notifies the drivers whether they are allowed to exit a street or not. In other words, vehicles are allowed to enter a street in both red and green ETL, but they are not allowed to exit the street for a long time in red ETL (while traffic is heavy in the subnetwork). The ETL gives a chance to relax traffic in a subnetwork and avoid traffic jam. The effectiveness of the proposed strategy is analysed and evaluated by a number of simulations on three-way grid networks. Two-way rectangular grid networks are modelled via a cell transmission model (CTM). The macroscopic fundamental diagram (MFD) and the number of jammed cells are compared with two state-of-the-art methods.
\end{abstract}

\section{Introduction}

Urban areas faced severe traffic congestion in recent decades, which causes serious problems such as increasing human travel time, fuel consumption, and air pollution. Traffic light is a popular equipment to control urban traffic. Phasing traffic light using a reasonable method reduces the delay time and increases the speed of vehicles and pedestrians on urban networks. The traffic signal strategies are mainly categorized as fixed-timed, coordinated traffic responsive [1], and predictive control strategies. Simple implementation and low management cost are two main reasons to use the fixed-timed strategy in lots of current urban traffic systems. The drawbacks of the fixed-timed model are that the resulting settings are based on the historical data rather than real-time data. SCOOT $[1,2]$ and SCATS $[1,3]$ are two well-known methods of the traffic responsive strategy that control traffic light based on the current traffic status. The shortcoming of this strategy is ignoring future traffic. The predictive control strategy is based on traffic forecasting models [4]. Ye et al. [1] studied the predictive control models in detail. The main idea of the model predictive control (MPC) is to transform a control problem into a model-based and online optimization problem with a rolling horizon. Although MPCbased coordination control approaches provide a good control performance in improving traffic efficiency and reducing fuel consumption and vehicle emissions, the key concern is the rapid increase of online computational complexity as the scale of the traffic networks increases [1]. The proposed strategies in this paper consider the current traffic status explicitly and the future traffic implicitly. The contributions of the paper are listed as follows:

(i) A new traffic light scheduling system is proposed based on eigenvector centrality, which manages 
traffic globally using local parameters. In the proposed method, we suggest a new representation of intersections based on matrix definition. In this way, all information of a junction is collected in a single data structure. We employ a well-known graph theory tool, namely eigendecomposition, to solve the traffic light scheduling problem, which is a new paradigm for this problem. The proposed traffic light system does not follow any phasing cycle and works based on the current and future traffic, dynamically. In this method, we employ a local parameter in our computations, but some shared features between adjacent junctions cause a global control and give the ability to predict future traffic.

(ii) A new type of traffic light is proposed based on the power of matrix, which is able to control the drivers' behaviour. This method predicts and prohibits the future traffic jams by forcing the drivers to change their direction to less-crowded areas.

This paper is organized as follows. Section 2 presents the literature review for the traffic light phasing strategies. Section 3 describes the proposed method. Section 4 presents the results of the simulations of the proposed method on some simulation scenarios and compares the results with two recent papers from literature. A discussion is provided in Section 5, and finally, a summary and conclusion is given in Section 6 .

\section{Literature Review}

Since traffic light is a popular equipment in all urban areas, many researchers have proposed methods to improve its phasing efficiency. In literature $[5,6]$, a traffic light phasing problem is formulated as a scheduling problem and aims to reduce the total waiting time over a given finite horizon. The most challenging part of a scheduling optimization problem is the real-time cases in which the scheduling is based on the current traffic status. Various optimization techniques have been proposed to solve the traffic light control problem. The state-of-the-art strategies proposed in the last few decades include MAXBAND [7, 8], TRANSYT (TRAffic Network Study Tool) [7, 8], SCOOT (Split Cycle Offset Optimization Technique) [9], OPAC (Optimized Policies for Adaptive Control) [10], PRODYN (Dynamic Programming) [11], CRONOS (ContRol of Networks by Optimization of Switchovers) [12], RHODES (real-time hierarchical optimized distributed effective system) [13], and SCATS (Sydney Coordinated Adaptive Traffic System) [3]. Some basic architectures for traffic light optimization are proposed based on genetic algorithm (GA) [14], cellular automata simulators (CAS) [15], and particle swarm optimization (PSO) [16].

Ye et al. [1] collected the methods which are presented to solve the problem of traffic light phasing problem from 1958 to recent years. The main scope of their study focuses on the model-based predictive control (MPC) over the last two decades. A number of recent models try to solve the traffic light phasing problem, using Petri net. Huang et al. [17] proposed a phasing method based on Petri net. Their approach classified intersections to 3 different types and set parameters for each type, separately. Their model has a good performance on traffic light phasing, but the process is too complex. Qi et al. [18] employed timed Petri net to model the drivers' behaviour and the conflicting traffic flow at intersections and divide them to two classes: nonjam-induced and jam-induced behaviour. Analysing drivers' behaviour can help to provide a right phasing scheme at intersections. They concluded that the jam-induced driving behaviour rate should be kept under 0.5 such that negligible delay is induced by such a behaviour.

Based on the literature, more than 20 self-adaptive traffic control systems have been developed for traffic light phasing problem, but less than $50 \%$ of them have been put into use [19]. The self-adaptive control systems can be divided to model-based (MBC) and model-free strategies. The modelbased self-adaptive control methods can be divided into two categories:

(1) Mathematical models

(2) Intelligent computing

The mathematical model-based control methods work based on the traffic status and the function of traffic forecasting module. Various mathematical MBC methods have been proposed in the literature, but the most important MBC models include the travel-time responsive (CTR) algorithm [20], arrival-discharge process [21], predictive model control [22], and storage-forward response control [23]. TRANSYT [24] and MULTIBAND [25] are two state-of-the-art MBC methods based on the comprehensive performance index and green wave band, respectively. The comprehensive performance index method concentrates on decreasing the delay, the number of stops, and the length of queues to increase the overall efficiency, while the green wave band method concentrates on increasing the number of nonstop vehicles in the network.

The artificial intelligence model-based control methods have been integrated to traffic light phasing strategies in recent decades. Among all artificial intelligence-based models, fuzzy logic, evolutionary algorithms, and neural network have received more attention from researchers [26-30]. Machine learning is also a paradigm to solve the problem of traffic light scheduling. Li et al. [31] proposed a method to solve the problem using reinforcement learning. Population-based metaheuristic algorithms have been employed for traffic light phasing optimization using GA [32] and the combination of GA and SA [33]. The STRAC by Spall and Chin [34], Markov process-based control by Hoogendoorn et al. [35], multiagent unsupervised flow response control model by Srinivasan et al. [36], and recurrent network architecture by Kosmatopoulos et al. [37] are the most important MBCs based on the neural network.

To the best of our knowledge, the concentration of all previous methods was on traffic status and not the traffic provider. In this note, the links that passes high traffic volume to a jammed intersection are called traffic jam providers (TJP). Uncontrolled drivers' behaviour causes high traffic volume movement toward specific intersection, which causes traffic jam. To find a reasonable traffic light 
phasing, we propose two strategies to control the traffic flow and drivers' behaviour, which are a combination of traffic responsive and predictive control strategies. The results indicate that the proposed strategies are able to distribute traffic uniformly, and the drivers redirect to less-crowded routes. Consequently, the traffic congestion and waiting time decrease and the vehicle speed increases in urban networks.

\section{The Proposed Traffic Light Control}

In this section, we present two strategies to manage the traffic in the urban area. In our settings, it is supposed that each traffic light has three lights which is shown in Figure 1. Since each light has red $(\mathrm{R})$, green $(\mathrm{G})$, and yellow $(\mathrm{Y})$ statuses, they have the capacity of sending 27 kinds of traffic light signals: RRR, RRY, RRG, RYR, RYY, RYG, RGR, RGY, RGG, YRR, YRY, YRG, YYR, YYY, YYG, YGR, YGY, YGG, GRR, GRY, GRG, GYR, GYY, GYG, GGR, GGY, and GGG. We also assume that all directions (north (n), east (e), south $(\mathrm{s})$, and west $(\mathrm{w})$ ) on a junction deploy two traffic lights.

(1) A set of typical traffic light to control an intersection

(2) A new class of dual mode traffic light (ETL), which indicates whether a vehicle can exit a street or not

For both ETL modes, the vehicles are allowed to enter a street, but when the ETL is red, the vehicles are not allowed to exit. To implement the ETL, we define the traffic jam provider, which is a street that transfers high volume traffic to an intersection. The red light of ETL on a traffic jam provider turns on. To propose our contribution, the following assumptions about a traffic network have been considered.

(i) The network entrance and exit models are known

(ii) The link turning ratios in the network are known

(iii) Each vehicle inside the network will leave the network, delayed only by traffic signals

3.1. Traffic Light Control Model. The proposed traffic light system is designed based on the eigenvector centrality. In graph theory, eigenvector centrality is a measure of the influence of a node in a network. Relative scores are assigned to all nodes in a network based on the concept that connections to high-scoring nodes are stronger than connections to low-scoring nodes. A high eigenscore means that a node is connected to many nodes with high scores $[38,39]$. In this paper, all essential information of an intersection is encapsulated in a matrix, which is called intersection matrix (IM). Each element of IM indicates the relation between two links of an intersection based on the waiting time and the number of vehicles on each link. However, we suppose that each link is equipped with two inductive loops to count the vehicle number at the entrance and exit. The model controls the traffic light colour dynamically, based on the current traffic status in three steps.

(1) As shown in Figure 2, each link has three relative destinations presented by $e \in\{l, r, s\}$ (l: left, $r$ : right,

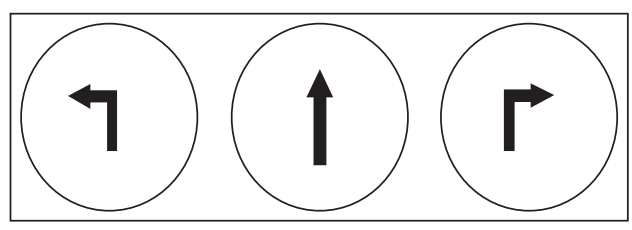

FIGURE 1: Each traffic light includes 3 lights with the capacity of sending 27 kinds of traffic light signals.

and $s$ : straight). The phasing strategy decides based on the traffic status of links and their relative destinations. For example, in Figure 2, links $h, f$, and $d$ are relative destinations for link $a$. Accordingly, the relation between each link and its relative destinations is calculated based on

$$
r_{i}=\frac{\left(W T_{i}+\eta\right) \times N v_{i}}{\sum_{e \in\{l, r, s\}} N v_{i}^{e}+1}+1
$$

where $\mathrm{WT}_{i}$ is the waiting time of all vehicles on link $i$, $N v_{i}$ is the number of vehicles on link $i$, and $N v_{i}^{e}(e \in\{l, r, s\})$ shows the vehicle number on left, right, or straight destinations of link $i$. In this equation, the traffic light supervisor has the chance to change the importance of the number of vehicles and waiting time using parameter $\eta$. For more values of $\eta$, the traffic light controller pays more attention to the number of vehicles on the link and vice versa. For example, the lower value of $\eta$ is more suitable for long links because lots of vehicles on the link might get the traffic light after 2 or 3 traffic light cycles. In these cases, the number of vehicles gives us the future status of traffic. There is a trade-off between selected parameter (the waiting time and the number of vehicles) in (1) that warranties the efficient traffic light scheduling. No vehicle waits for a long time, and the overall waiting time and travel time will be minimized. The value of $r_{i}$ is bigger if the vehicle waiting time and the number of vehicles on link $i$ is higher and the number of vehicles on destination links is lower.

(2) Usually, an intersection is connected to four links with 12 directions as shown in Figure 2. Therefore, an intersection matrix $\left(\mathrm{IM}_{12 \times 12}\right)$ is generated as the following:

$$
\operatorname{IM}(i, j)=\frac{r_{i}}{r_{j}}, \quad i \neq j .
$$

The IM includes the relative traffic status between all pairs of origin and destination on an intersection. More $\operatorname{IM}(i, j)$, heavier relative traffic on link $i$, and less $\operatorname{IM}(i, j)$, heavier relative traffic on link $j$.

(3) In the last step, the traffic light mode (green or red) is selected for each link. To calculate the priority of 


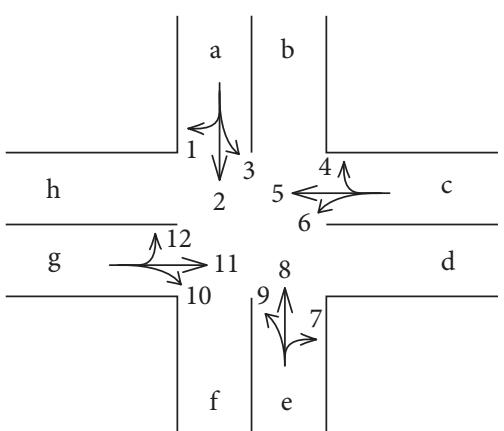

FIgURE 2: A typical intersection with four connected links is shown in this illustration. Links (a), (c), (e), and (g) are inflow links of this intersection. Drivers on each link have three choices to continue their path: left, right, and straight forward. However, there are four inflow links, each of which has three possible destination links at intersection. Accordingly, an intersection with four connected link provides 12 directions.

streets for the green mode, the eigenvector centrality of the IM is calculated. Eigendecomposition is a wellknown mathematical tool in all programming languages which is shown in $\mathrm{fd} 3$.

$$
\mathrm{IM} \times V=D \times V .
$$

Each column of $V$ is an eigenvector of IM, and values on the main diameter of $D$ are eigenvalues of IM. Eigenvectors are orthonormal vectors in $(0,1)$. The eigenvector with the biggest eigenvalue is used to calculate eigenscores of IM. The values on this eigenvector are the eigenscores of intersection's links. Since IM $\in \mathbb{R}^{12 \times 12}$, the eigenvector of IM is vector $E S \in \mathbb{R}^{12 \times 1}$. Therefore, the eigendecomposition assigns a score to each direction of a junction. Eventually, the priority of each link is calculated based on the summation of its directions' eigenscores as the following:

$$
S_{X}=\mathrm{ES}_{X}^{r}+\mathrm{ES}_{X}^{l}+\mathrm{ES}_{X}^{s}, \quad X \in\{N, S, E, W\},
$$

where $X$ is a direction of intersection links: north $(\mathrm{N})$, south $(\mathrm{S})$, east(E), and west (W). $S_{X}$ is the score in direction $X$, and $\mathrm{ES}_{X}^{r}, \mathrm{ES}_{X}^{l}$, and $\mathrm{ES}_{X}^{s}$ are the eigenscores of link $X$ to relative destinations $r, l$, and $s$, respectively. The street with the biggest $S_{X}$, in addition to its collision-free routes, is set to the green phase. The collision-free routes are those without collision with the selected link.

The other traffic lights of the same intersection are set according to their eigenscore as the following:

(i) The other links are examined in the descending order of their eigenscore. If a direction has collision with green directions, it receives red status.

(ii) Otherwise, it receives green status.

The proposed traffic light control system does not follow any phasing cycle. A phasing scheme is valid on junction until its condition is true (while its eigenscore ranking does not change). While the IM is changing regularly, eigenscores are calculated in real time. Therefore, the current phasing changes when the intersection's traffic status changes. A minimum duration time is defined for each phase, but the phase period time is decided based on the intersection traffic status. The existence of $\mathrm{WT}_{i}$ to calculate the green phase priority prohibits the algorithm to give a very long duration to a phasing scheme. In the proposed strategy, the priority of links for the green phase depends on three parameters:

(1) The accumulative waiting time of the link

(2) The occupancy on the link

(3) The occupancy of its destination links

As can be seen in Figure 3, two adjacent junctions ( $n$ and $m$ ) have a shared link $(E)$, which is appeared in (1) as $N v_{i}^{e} . E_{D}$ is a local destination of intersection $m$ and a local origin of intersection $n$, and $\mathrm{E}_{\mathrm{U}}$ is the destination and origin of intersections $n$ and $m$, respectively. To calculate $\mathrm{IM}_{\mathrm{m}}$ and $\mathrm{IM}_{\mathrm{n}}$, parameters $N v_{n}^{e}$ and $N v_{m}^{e}$ appears in $r_{3}^{n}, r_{7}^{n}, r_{11}^{n}, r_{1}^{m}, r_{5}^{m}$, and $r_{5}^{m}$ (the subscript numbers are equivalent to numbers in Figure 2). Accordingly, any traffic light scheduling for node $m$ affects the traffic status of its neighbours (include $n$ ) and neighbours of neighbours. Such internal dependency between adjacent traffic lights (using common variables) led to an implicit global control over a traffic network.

3.2. Exit Status Traffic Light (ETL). The proposed traffic light strategy, which is presented in Section 3.1, manages the traffic jam problem by distributing the traffic uniformly over the network, but the traffic jam is inevitable if traffic volume exceeds the network capacity. None of the current phasing strategy is able to handle traffic of a network which is full of vehicles. In the other words, each traffic light phasing strategy increases the network capacity, but no strategy is able to manage the traffic in an overloaded traffic network. To cope with this problem, we propose another strategy, which is named ETL, to control the drivers' behaviour and avoid vehicle overload. To continue, we need to define two expressions:

(1) Jammed node $(J)$ : an intersection in the network, which is involved in a heavy traffic

(2) Jam provider $\left(p_{d}^{i}\right)$ : a node of network with distance $d$ from $i$ and transfers a high volume traffic to jammed node $i$

The proposed ETL, as shown in Figure 4, is a dual-mode traffic light that is hanged on the entrance of a link to show the exit status. The entrance to a link is allowed in both red and green ETLs, but the exit is prohibited in the case of red ETL. The main idea of ETL comes from the fact that drivers usually have a static information about an urban network such as the shortest path and freeways. The dynamic information of the urban network such as jammed intersection, the noisiest path, current flow speed, and the whole traffic status are not known for drivers without special equipment. Therefore, they usually select the best path based on their static knowledge about the network. The ETL helps drivers to change their routes by informing traffic jam or heavy traffic on their future path. Using ETL, we can directly 


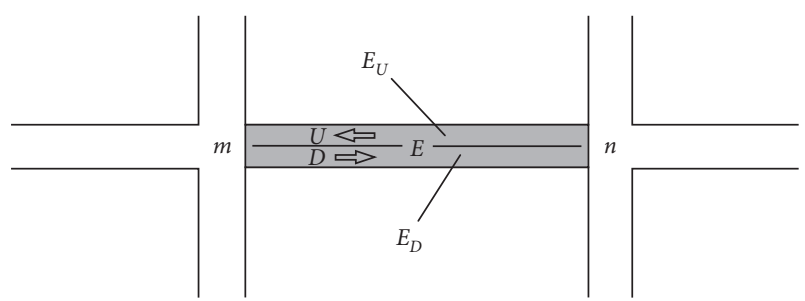

FIgURE 3: This image indicates a shared link between two intersections. Link $E$ is the shared link for adjacent junctions $n$ and $(m)$. This common link is presented in (1) as $N v_{i}^{e}$. ED is the destination link of intersection $m$ and origin link of intersection $(n)$, but EU is the destination and origin of intersections $n$ and $(m)$, respectively. The intersection matrices of junctions $m$ and $n$, IMm and IMn, have two common parameters $N v_{n}^{e}$ and $N v_{m}^{e}$ in $r_{3}^{n}, r_{7}^{n}, r_{11}^{n}, r_{1}^{m}, r_{5}^{m}$, and $r_{5}^{m}$.

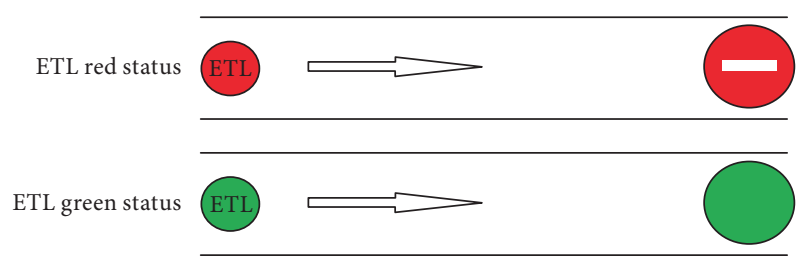

FIgURE 4: The ETL traffic light which is a dual mode traffic light that is set at the entrance of a link to show the exit status of the link is shown. The entrance to a link is allowed in both cases of red and green ETL, but the exit is banned in the case of red ETL.

control the behaviour of drivers to increase the network efficiency and decrease the travel time and emissions. The ETL uses the chain-based nature of traffic entity, which is formulated as follows:

$$
\tau_{i}=\sum_{k \in K^{-1}} \alpha_{1}^{k} \tau_{k}+\sum_{k \in K^{-2}} \alpha_{2}^{k} \tau_{k},
$$

where $\tau_{i}$ is the traffic volume at node $i, K^{-j}$ is the set of nodes with distance $j$ to node $i$, and $\alpha_{j}^{k}$ is the portion of traffic volume that comes from node $k$ to node $i$. The ETL of distinguished traffic jam providers gets the red status. The ETL includes two steps:

(1) Estimating the traffic jammed intersections

(2) Detecting traffic jam providers for jammed intersections and turning the red ETL on

Both these steps are explained in the following sections.

3.2.1. Predicting Traffic Jam. To predict jammed intersections, (6) is proposed:

$$
\mathrm{FC}=\text { Row_sum }\left(\sum_{i=1}^{l} \omega_{i} A^{i}\right),
$$

where $\omega_{i}$ is the weighting scheme of the model, $A$ is the traffic flow matrix, and Row_sum (B) $\in \mathbb{R}^{n}$ is a rowwise summation of the matrix $B \in \mathbb{R}^{(n, m)}$. The traffic flow matrix $(A)$ indicates the traffic flow on each link of matrix $A$. Each element of $\omega_{i} \times A^{i}$ presents the traffic volume that transfers between a pair of networks' node. The $i^{\text {th }}$ power of $A$ shows the traffic volume that is moving between nodes with distance $i$. However, FC is the summation of $1^{\text {st }}, 2^{\text {nd }}, \ldots, l^{\text {th }}$ power of $A$, which includes traffic volume between all O-D ${ }^{8}$ pairs with maximum distance $l$. Suppose $i$ and $j$ are two intersections of a traffic network. To compute $A_{(i, j)}^{n}$, the mathematical expression $A_{(i, 1)} \times A_{(1,2)} \times A_{(2,3)} \times \cdots \times A_{(n, j)}$ is calculated for all existing routes (with length $n$ ) between $i$ and $j$, where $A_{(p, q)}$ is the traffic volume that is moving from node $q$ to node $p$. The more traffic volume between $j$ and $i$, the bigger $\mathrm{FC}_{(i, j)}$. One hundred percentage of vehicles on $\mathrm{FC}_{(i, j)}$ move from $j$ to $i$, when $n=1$, because there is only one destination between two neighbour nodes. By increasing the value of $n$, the number of intermediate destinations increases. However, for bigger $n$, the lower portion of vehicles on $j$ moves toward $i\left(\omega_{n} \leq \omega_{n-1} \leq \cdots \leq \omega_{2} \leq \omega_{1}\right) . \omega_{i}$ is defined to set the portion of traffic volume that move between intersections with different distances, obtained using census. Accordingly, row $i$ of $\sum_{i=1}^{l} \omega_{i} \times A^{i}$ includes the traffic volumes that come from all network's nodes to node $i$. Hence, the $i^{\text {th }}$ component of FC indicates the number of vehicles travelling to node $i$. By setting a threshold $(\theta)$ based on network capacity, traffic jam can be predicted simply. A traffic jam is occurred on node $i$, if $\mathrm{FC}_{i}>\theta$. To this step, we can predict the future traffic jams over the network. The next step is detecting the intersections that pass high volume traffic to node $i$ (Traffic jam providers) and ban their directions to node $i$ using ETL. The ETL is a predictor that is able to forecast future traffic, in addition to traffic jam providers.

3.2.2. Detecting Traffic Jam Providers. After predicting the traffic jam, the next step is finding traffic jam providers using the following equation:

$$
\mathrm{TR}^{d}=\sum_{i=1}^{d} \omega_{i} A^{i}
$$

All variables are similar to (6). Parameter $d$ sets the distance of the traffic jam provider to current intersection, i.e., $d=2$ indicates that neighbours with distance less than 3 are involved in the calculation. $\operatorname{TR}_{(i, j)}^{d}$ indicates the volume of vehicles that node $j$ transfers to node $i$ with maximum distance. The biggest elements on row $i$ transfer more traffic to node $i$, which is distinguished as the traffic jam provider of node $i$. By activating red ETL on traffic jam providers, the traffic will be relaxed on node $i$, and the future traffic jam will not happened. In general, the ETL red mode is a long phase, which is activated until traffic get relaxed on the specific zone. The ETL has some considerable benefits that include the following:

(1) The ETL controls the behaviour of drivers to select their path.

(2) The ETL has simple implementation.

(3) Looking for parking is one basic reason that makes traffic flow slower, which resulted in traffic jam. Since the ETL is a long traffic phase, the link with red status can be used as temporal parking to reduce the 
parking problems, specifically in central regions of cities.

(4) The ETL can be more effective by sustainable transportation systems such as shared bicycle.

\section{Experimental Result}

We propose two new traffic light strategies based on the power of matrix. The effectiveness of the proposed strategies are evaluated by employing Python and SUMO through simulation. SUMO is an open source, highly portable, microscopic, and a continuous traffic simulation package, designed to handle large urban networks. First, we built twoway rectangular grid networks based on a cell transmission model (CTM) [41, 42]. Through the simulation of our designed model, we identify the influences of some important parameters, i.e., route-changing behaviours of drivers, the trade-off between waiting time and traffic flow in (1), and the inflow of the traffic network on the traffic congestion. Table 1 describes the symbols, used in the following content.

4.1. Cell Transmission Model. CTM is proposed by Daganzo $[41,42]$ to simplify the solution scheme of the Lighthill-Whitham-Richards (LWR) model [2, 43] by adopting the following relationship between traffic flow $q$ (vehicles per hour) and density $k$ (vehicles per lane-mile) as shown in Figure 5:

$$
q=\min \left\{v k, q_{\max }, w\left(k_{j}-k\right)\right\}, \quad 0 \leq k \leq k_{j},
$$

where $q_{\max }, K_{j}, v$, and $w$, respectively, denote the maximum flow, jam density, flow, and backward shock wave. The negative $w$ means the network is going to be jammed. Higher $v$ causes the smooth flow in the network and the maximum $v$ is the free flow. The network tolerates a maximum traffic flow $\left(q_{\max }\right)$, and the traffic jam occurs if the number of vehicles increases. In this paper, we use the following CTM [44]:

$$
\begin{aligned}
y_{i}(t) & =\min \left\{n_{i-1}(t), Q_{i}(t), w\left(N_{i}(t)-\frac{n_{i}(t)}{v} k\right)\right\}, \\
n_{i}(t+1) & =n_{i}(t)+y_{i}(t)-y_{i+1}(t),
\end{aligned}
$$

where $y_{i}(t)$ is the number of vehicles that flow into cell $i$ during time interval $t, n_{i}(t)$ is the number of vehicles in cell $i$ before $t, N_{i}(t)$ denotes the maximum number of vehicles that can be contained in cell $I$ during $t$, and $Q_{i}(t)$ denotes the inflow capacity in cell $i$ during $t$.

4.2. Traffic Network. To evaluate the proposed method and make a justified comparison, two networks are deployed from literature $[44,45]$. The networks are squared two-way grid networks similar to Figure 6 with different sizes: $8 \times 8$, $5 \times 5$, and $4 \times 4$. All boundary nodes are both origins and destinations. The turning rate for each vehicle is presented by $P_{l}, P_{r}$, and $P_{s}\left(\right.$ where $\left.P_{l}+P_{r}+P_{s}=1\right)$ for left, right, and
TABLE 1: Symbols used in this paper.

\begin{tabular}{lc}
\hline Symbols & Meaning \\
\hline$v$ & Free flow speed (miles per hour) \\
$w$ & The speed of all backward moving waves \\
(miles per hour)
\end{tabular}

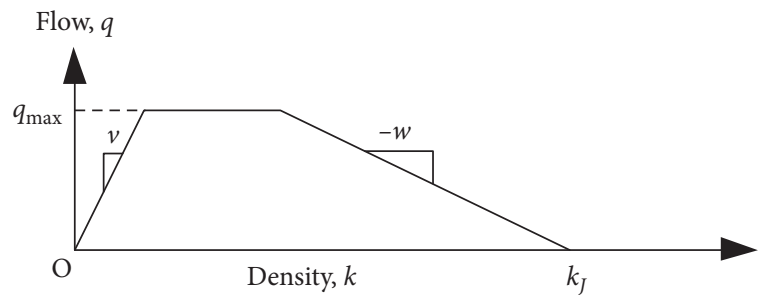

FIGURE 5: Flow-density relationship for the generalized CTM [40]. This diagram indicates the trade-off between network flow and vehicle density on the network. If the density exceeds from a threshold, the traffic jam is avoidable. $q_{\max }, K_{j}, v$, and $w$, respectively, denote the maximum flow, jam density, flow, and backward shock wave. The negative $w$ means the network is going to be jammed. Higher $v$ causes the smooth flow in the network, and the maximum $v$ is the free flow. The network tolerates a maximum traffic flow $\left(q_{\max }\right)$, and the traffic jam occurs if the number of vehicles increased.

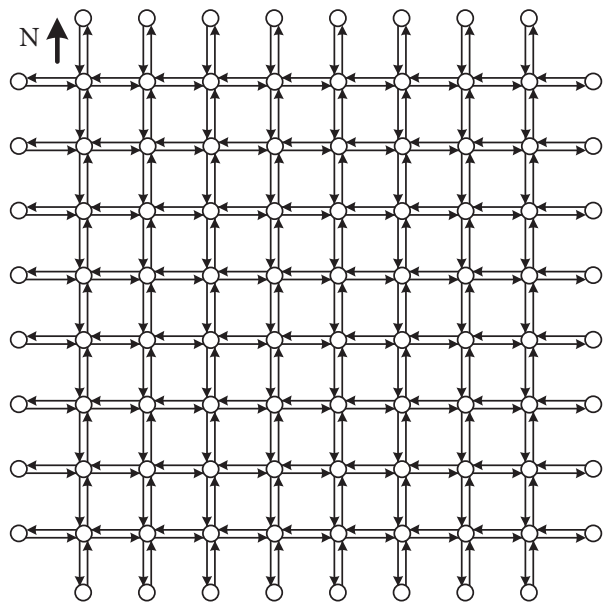

Figure 6: An $8 \times 8$ two-way grid network. All boundary nodes are both origins and destinations.

straight directions, respectively. The networks features are presented in Table 2.

In this paper, we consider 6 simulation scenarios with different traffic demands for the simulation process. We simulate the peak hour situation in the scenario 1 with an increasing demand from the beginning of the simulation and then decreasing demand toward the end. The oversaturated 
TABLE 2: The properties of networks.

\begin{tabular}{lccccccc}
\hline Network & Link length $(\mathrm{m})$ & No. of lanes & No. of O-D & Vehicle length $(\mathrm{m})$ & Flow capacity & Time interval (s) & Simulation time $(\mathrm{s})$ \\
\hline $4 \times 4[45]$ & 500 & 3 & 16 & 7 & 2000 (veh/h/lane) & 5 & 5400 \\
$5 \times 5$ & 675 & 2 & 20 & 7.5 & 1800 (veh/h/lane) & 5 & 1800 \\
$8 \times 8[44]$ & 675 & 2 & 32 & 7.5 & 1800 (veh/h/lane) & 5 & 10000 \\
\hline
\end{tabular}

traffic condition is simulated using increasing traffic demand in scenarios 2,4 , and 5 . The sudden change in the traffic flow is simulated using an incident in the network in scenario 3. The unequilibrated traffic in the network with heavy traffic on some routes of the network is in scenario 6. The traffic demand is $\Gamma$ vehicles per interval for each origin, which is assumed to be equal for all source nodes that are presented in Table 3. The flow proportions for all directions are $P_{l}=0.25$, $P_{r}=0.25$, and $P_{s}=0.5$. The initial network is empty, and some time intervals are required to allow the system to stabilize.

4.3. Results of the Simulation Scenario 3. In this simulation, we evaluate the proposed traffic light scheduling model using a CTM configuration. To have a justified comparison, the CTM parameters are taken from the study by Qi et al. [44] as shown in Table 4. At the first analysis, we survey the effect of $\eta$ in (1) in the proposed model. For the bigger value of $\eta$, the model gives more weight to inflow traffic. Figure 7 indicates the simulation results with different values of $\eta$. However, we set $\eta=2$ in configuration of the model for the later experiments. As the other analysis in this section, we study the effect of the traffic demand $\Gamma$ on jam formation and dissipation to compare the result with conventional fixed traffic lights and reported results by Stevanovic [19]. Figures 8 and 9 indicate the throughput and number of jammed cells during the simulation using traffic demands $(\Gamma=0.5,0.8,1,1.5,2)$, respectively. The throughput indicator shows how a network is able to respond to the traffic status and increase the traffic outflow. Figures 8(b) and 9(b) clearly show that, using the proposed strategy, the network throughput is stable even in heavy traffic. Figure 9(c) presents the reported results by Qi et al. [44] with the same setting. In the simulation of scenario 3 , an incident is start from step 300 to 1000 . By comparing the results, we can see that the proposed method increases the network efficiency even when a traffic incident is simulated in the network. We experiment higher traffic demand, and the results show better control by the proposed method, even on heavier traffic condition.

4.4. Results of Simulation Scenarios 1, 2, 4, and 5. Through this section, the results of simulation scenarios 1,2 , 4 , and 5 in a $4 \times 4$ network is presented. The parameters of scenarios 1 and 2 are taken from the seminal work of Yan et al. [45]. The simulation scenarios 4 and 5 are simulated by higher traffic demands, with two and three times more traffic demand than scenario 2, respectively. The reported results by Yan et al. [45], which include 5 run with different random seeds (MFD1, ..., MFD5), are presented in Figure 10. MFD [46] is a type of traffic flow fundamental diagram that relates space-mean flow, density, and speed of an entire network with $n$ number of links. The experiments are illustrated in Figure 11 and show that the better efficiency of the proposed method is in comparison to the proposed method by Yan et al. [45]. The results of the CTM model are presented in Figure 12. All illustrations indicate the ability of the proposed method to manage heavy traffics.

4.5. Results of Simulation Scenario 6. In simulation scenario 6 , a uniform traffic is running over the network, except a vertical route and a horizontal route with 20 times more traffic volume than other links. Heavy traffic on $3^{\text {rd }}$ vertical and $3^{\text {rd }}$ horizontal routes on a $5 \times 5$ grid network cause heavy traffic in the centre of the network. The simulation scenario 6 is designed to study the effects of ETL using fixed traffic lights. In this scenario, we compare the network traffic status with and without ETL strategy. Figure 13 indicates the number of jammed cells and waiting time of all vehicles in a $5 \times 5$ network with fixed time traffic lights. Figure 14 shows the result of activating two ETLs in the network. The ETL aims the following improvements in the network:

(1) About 10\% improvement in the number of jammed cells by distributing traffic over the network

(2) Decrease the cumulative waiting time

(3) Free a number of streets in the network that can be used as temporal parking while the ETL is activated

\section{Discussion}

We analyse the ability of the proposed method to control the drivers' behaviour and traffic dissipation through this section.

5.1. The Control on Drivers' Behaviour. We proposed two strategies, both of them control drivers' behaviour to relax traffic. Suppose a vehicle starts from origin $O$ to destination $P$ in Figure 15. The grey links indicate heavy traffic. The proposed method gives more green cycles to vertical routes than horizontal ones. Setting more green cycles to horizontal routes does not decrease the traffic volume because vehicles cannot move from $O$ to $a$ and from $a$ to $b$. In the other words, the capacity of destinations on horizontal routes is full. However, green cycle time for horizontal routes increases the waiting time of vehicles on vertical routes. Hence, the proposed strategy puts two options in front of the drivers: changing their routes or waiting for a long time. The drivers probably prefer to change their routes rather than waiting for a long time, which causes a uniform traffic distribution over the network and prevents traffic jam appearance. 
TABLE 3: The traffic demand for each simulation scenario.

\begin{tabular}{lccccc}
\hline Simulation time $\left(10^{3} \mathrm{~s}\right)$ & $0-0.9$ & $0.9-1.8$ & $1.8-2.7$ & $2.7-3.6$ & $3.6-4.5$ \\
\hline Simulation scenario 1 $(4 \times 4)[44]$ & 1000 & 1800 & 2000 & 2500 & 2000 \\
Simulation scenario 2 $(4 \times 4)[44]$ & 2000 & 2200 & 2400 & 2600 & 2800 \\
Simulation scenario 3 $(8 \times 8)[45]$ & 1920 & 1920 & 1920 & 1920 & 1920 \\
Simulation scenario 4 $(4 \times 4)[44]$ & 4000 & 4400 & 4800 & 5200 & 5600 \\
Simulation scenario 5 $(4 \times 4)[44]$ & 6000 & 6600 & 7200 & 7800 & 8400 \\
Simulation scenario 6 $(5 \times 45)$ & 6900 & 6900 & - & - & - \\
\hline
\end{tabular}

TABLE 4: CTM parameters [44] for the scenario 3.

\begin{tabular}{lc}
\hline Parameters & Value \\
\hline $\begin{array}{l}\text { The length of each time interval } \\
\text { Jam density }\end{array}$ & 133 vehicles/ $\mathrm{km} /$ lanes (i.e., $7.5 \mathrm{~m}$ for every vehicle in each lane) \\
$\begin{array}{l}\text { Number of lanes } \\
\text { Flow capacity }\end{array}$ & $\begin{array}{c}\mathrm{km} / \mathrm{h} \text { (i.e., } 15 \mathrm{~m} / \mathrm{s} \text { ) } \\
\text { The number of cells of each link }\end{array}$ \\
\hline
\end{tabular}

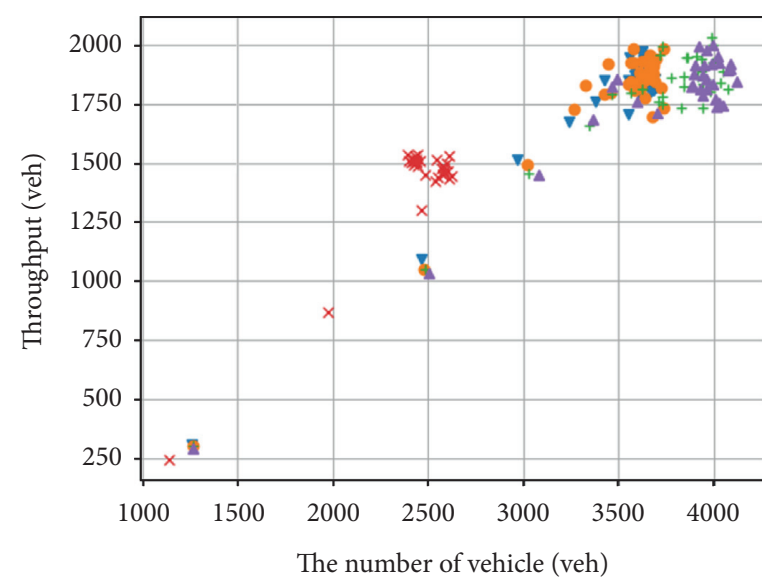

$$
\begin{array}{rlrl}
\nabla & =0.5 & \times & \times 2 \\
\eta & =1 & & \Delta \eta=3 \\
+\eta & =1.5 &
\end{array}
$$

(a)

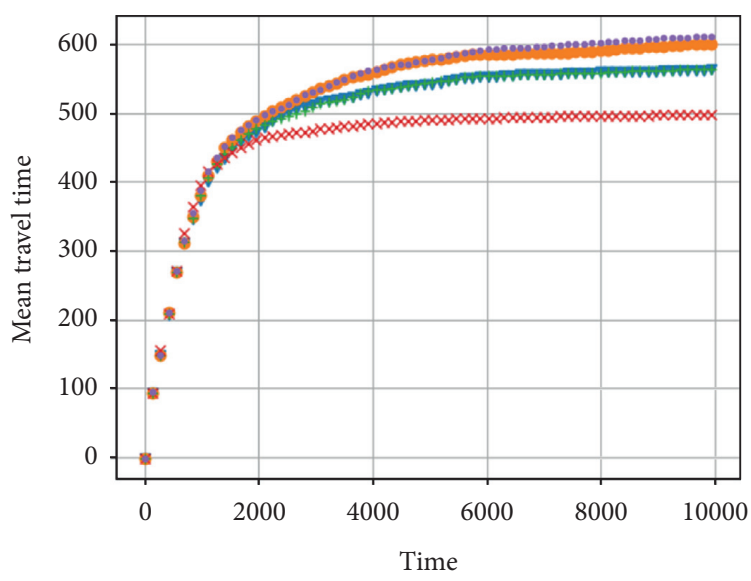

$$
\begin{array}{rlrl}
\nabla \eta & =0.5 & \times \eta=2 \\
\eta & =1 & & \times \eta=3 \\
+\eta & =1.5 &
\end{array}
$$

(c)

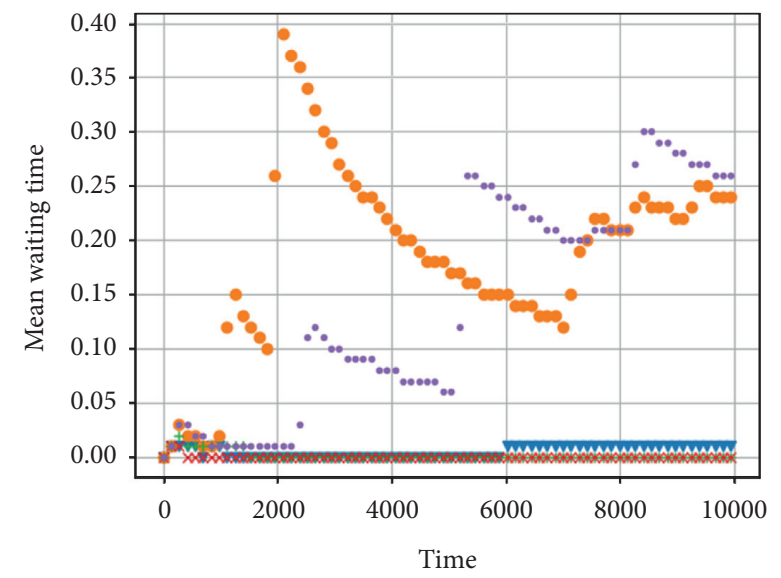

$$
\begin{array}{ll}
\nabla \eta=0.5 & \times \eta=2 \\
\eta=1 & \times \eta=3 \\
+\eta=1.5 &
\end{array}
$$

(b)

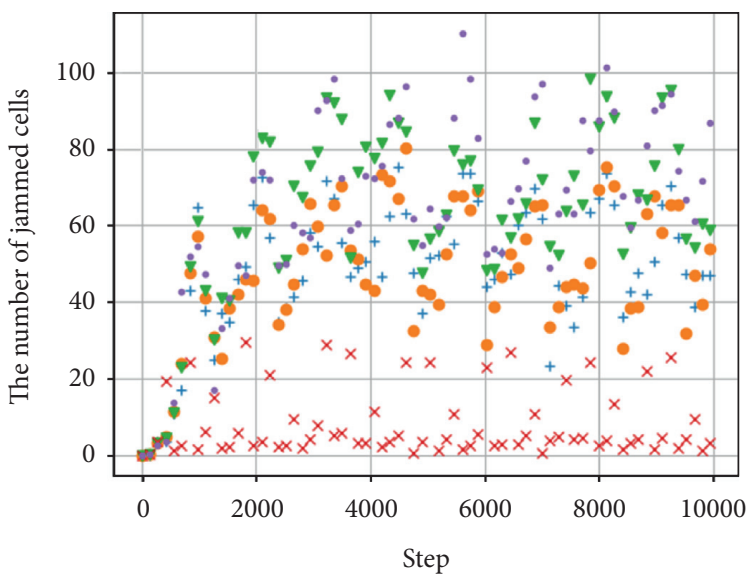

$$
\begin{array}{ll}
+\eta=0.5 & \times \eta=2 \\
\vee \eta=1 & \\
\nabla \eta=1.5 &
\end{array}
$$

(d)

Figure 7: The network evaluation using different values of $\eta=0.5,1,1.5,2,3$ of an $8 \times 8$ network with one strategy for $\Gamma=1$. These images compare the simulation results of different $\eta$. As can be seen, $\eta=2$ is the well configuration of our model. (a) The network throughput. (b) The average waiting time. (c) The average travel time. (d) The number of jammed cells. 

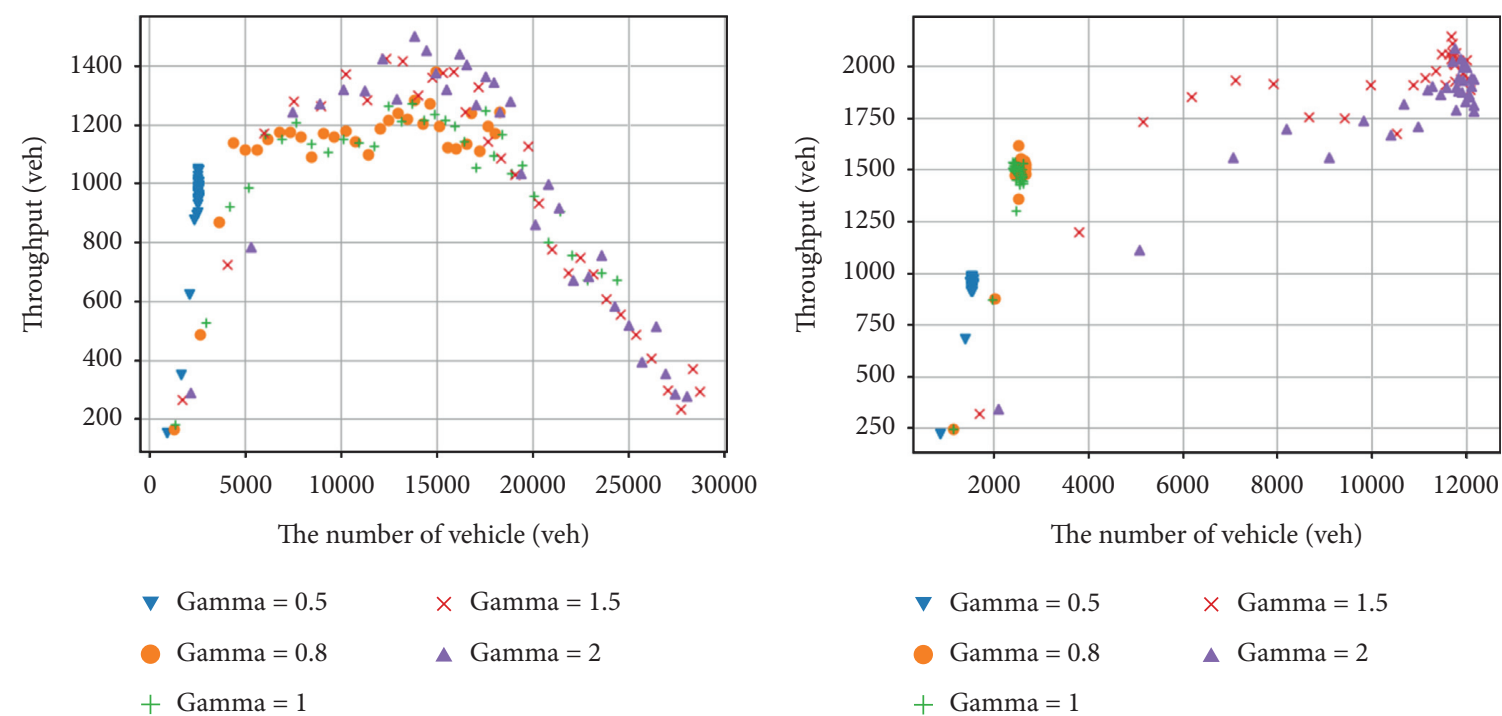

(a)

(b)

FiguRE 8: The throughput of an $8 \times 8$ network with one strategy and fixed phasing time for $\Gamma=0.5,0.8,1,1.5,2$. The throughput indicates the ability of method to manage the traffic, while it has an increasing traffic trend. (a) Fixed time traffic light. (b) The proposed traffic light phasing without ETL.

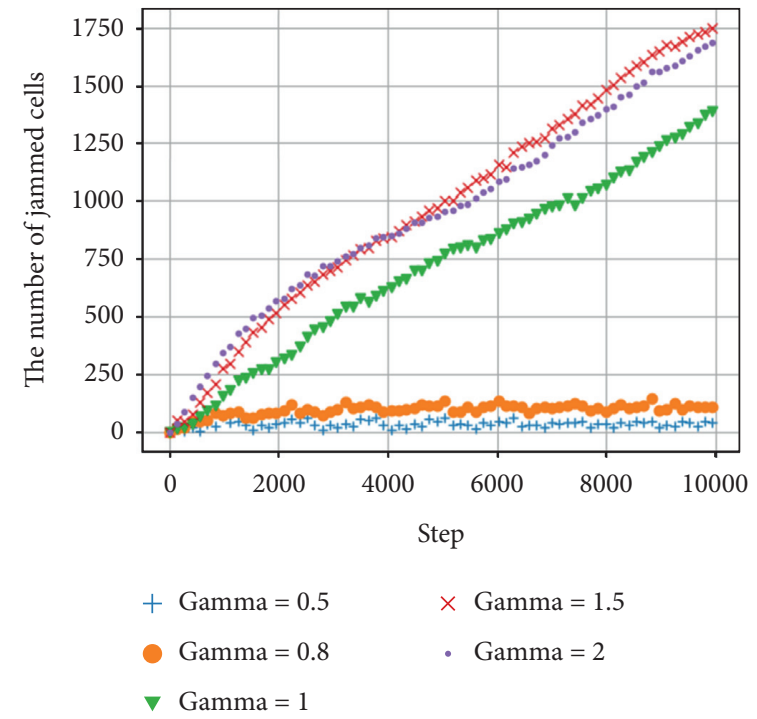

(a)

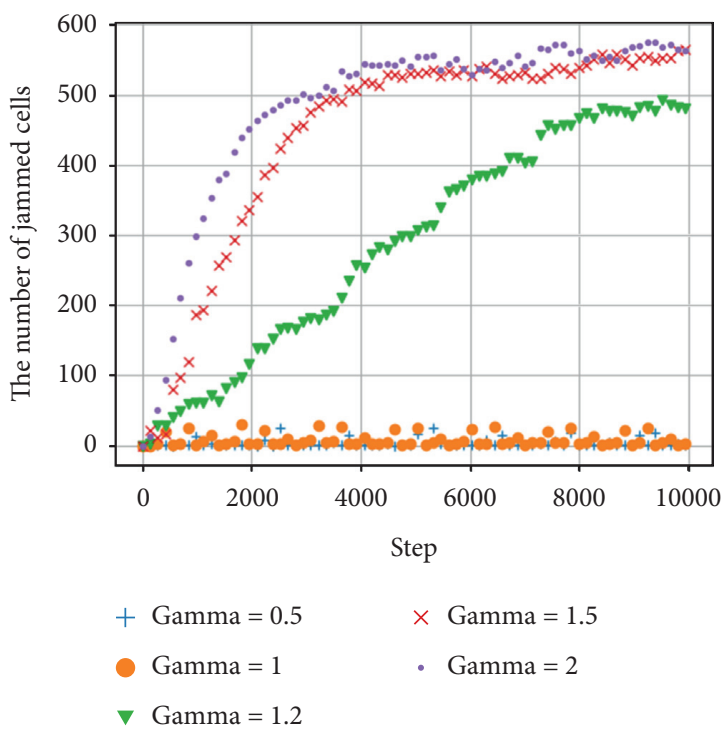

(b)

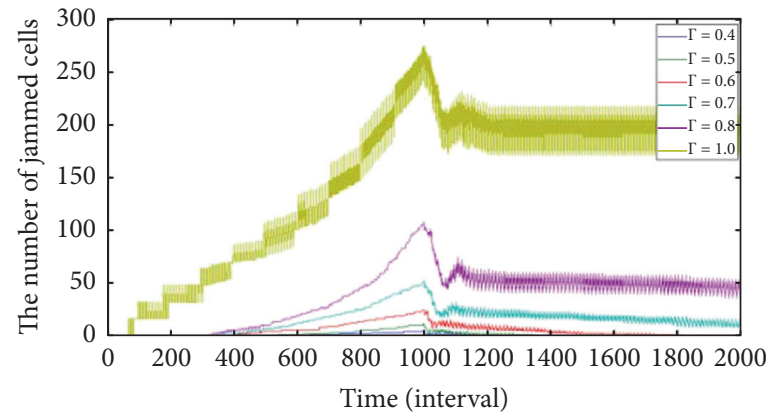

(c)

FIgURE 9: The number of jammed cells of an $8 \times 8$ network with one strategy and fixed phasing time for $\Gamma=[0.5,1,1.2,1.5,2]$. The method is successful to handle the traffic, if the number of jammed cells is low. (a) Fixed time traffic light. (b) The proposed traffic light phasing without ETL. (c) The proposed traffic light phasing by Qi et al. [44]. 


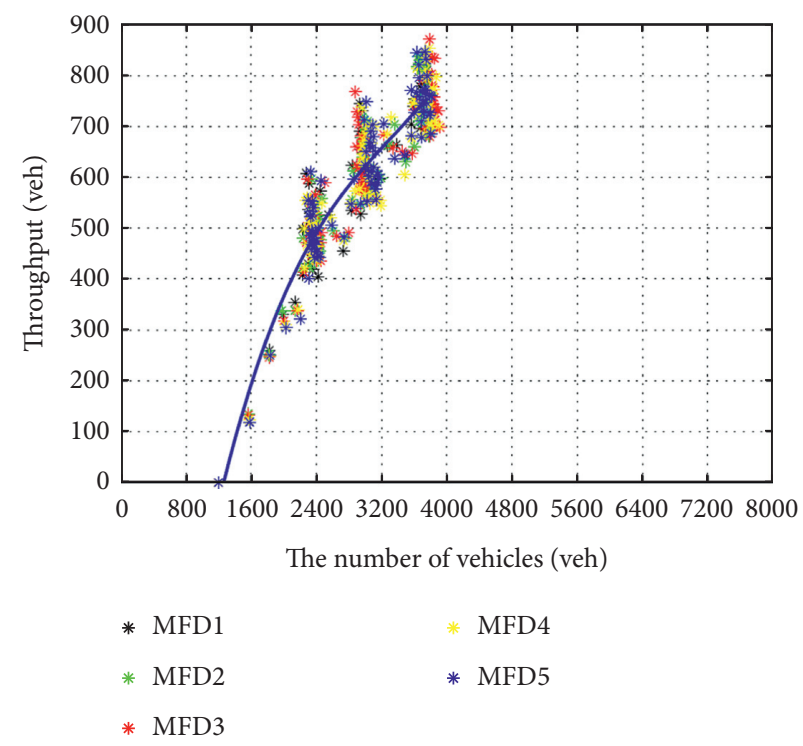

(a)

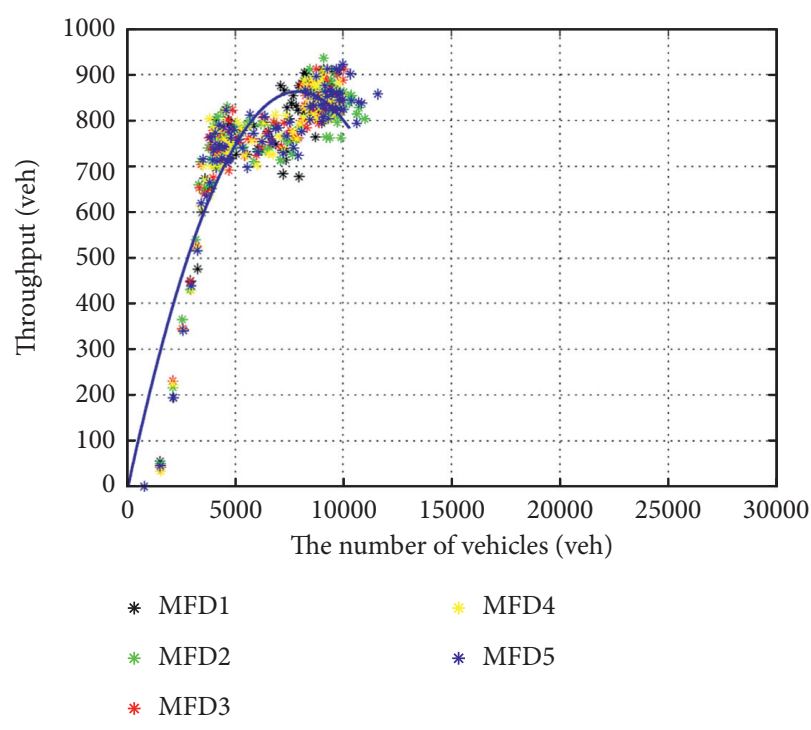

(b)

Figure 10: The throughput of a $4 \times 4$ network reported by Yan et al. [45] for scenarios 1 and 2 . This result is presented here to compare with the proposed method. (a) The proposed traffic light phasing reported for the scenario 1 . (b) The proposed traffic light phasing reported for the scenario 2 .

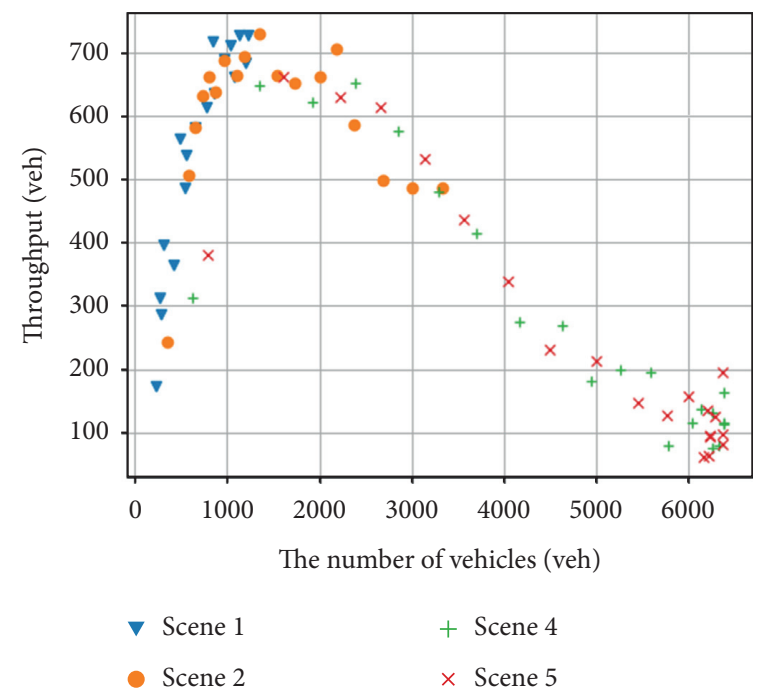

(a)

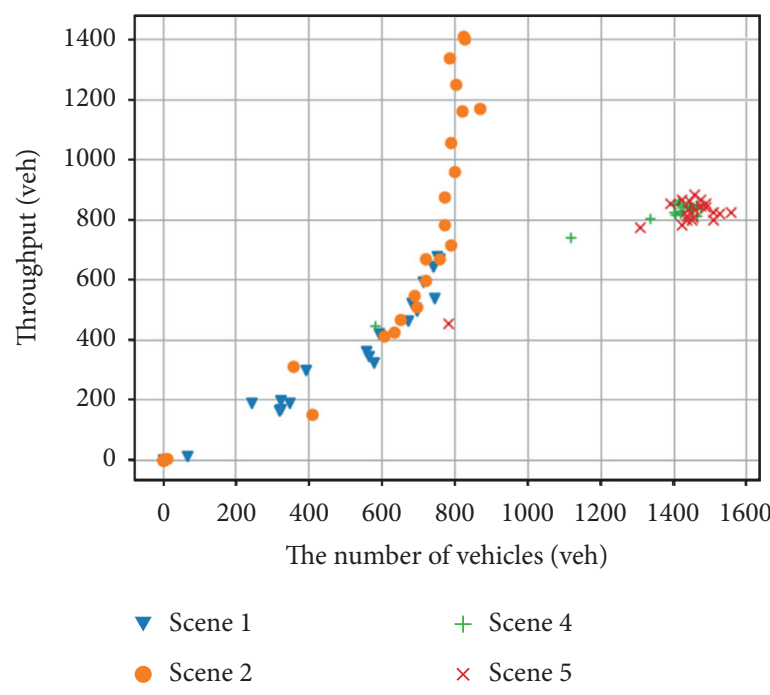

(b)

Figure 11: The throughput of a $4 \times 4$ network with one strategy and fixed phasing time for scenarios $1,2,4$, and 5 . By comparing the results with Figure 10, it can clearly be seen that the proposed method is more efficient than the results of Yan et al. [45]. It is also able to control the traffic in very heavy traffic like scenarios 4 and 5. (a) Fixed time traffic light. (b) The proposed traffic light phasing without ETL.

The concentration of the ETL is more on controlling the drivers' behaviour rather than satisfying their demands. The main goal of traffic light scheduling systems is increasing the network capacity to have more throughput, but they fail in the overloaded networks. To solve the problem, the ETL strategy is introduced to control the incoming traffic flow to a region or a subnetwork. Figure 16 illustrates a sample network with a traffic jam on junction $B$. As can be seen, link numbers 1, 2, and 3 are traffic jam providers with distance two, and link number 6 is the traffic jam provider with distance one for junction B. Using the ELT, the entrance to link number 6 is prevented until the traffic be dissipated. Using this strategy, drivers on links 1, 2, and 3 change their route to use alternative paths such as links 5 and 7. Accordingly, the traffic is distributed uniformly over the network to satisfy the user equilibrium principle. 


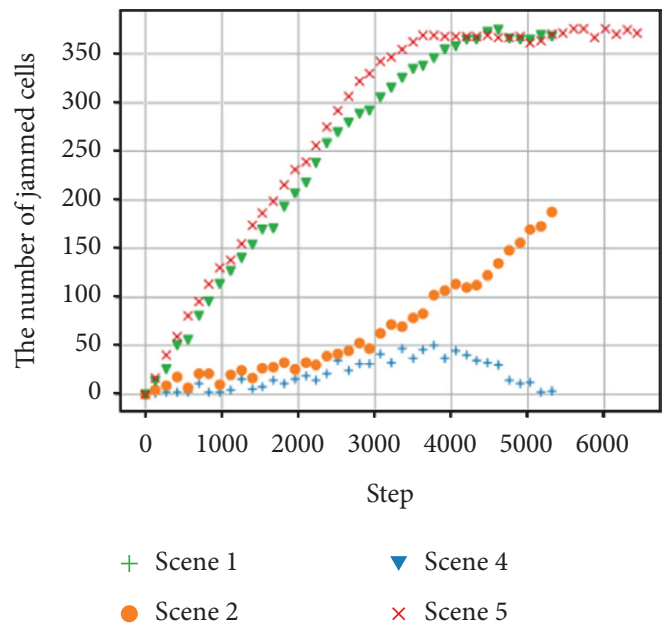

(a)

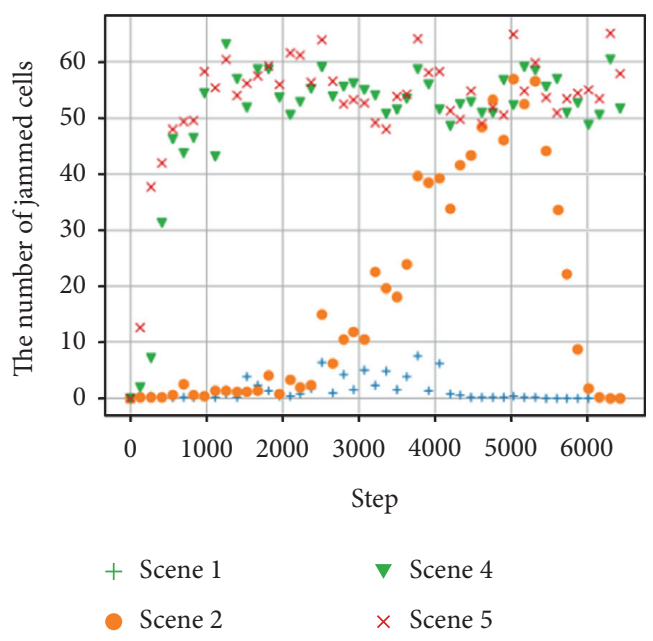

(b)

Figure 12: The number of jammed cells of a $4 \times 4$ network with one strategy and fixed phasing time for scenarios 1, 2, 4, and 5 . By comparing the results with the fixed time traffic light, we can see that the proposed method manage the network number of jammed cells better in heavy traffic. (a) Fixed time traffic light. (b) The proposed traffic light phasing without ETL.

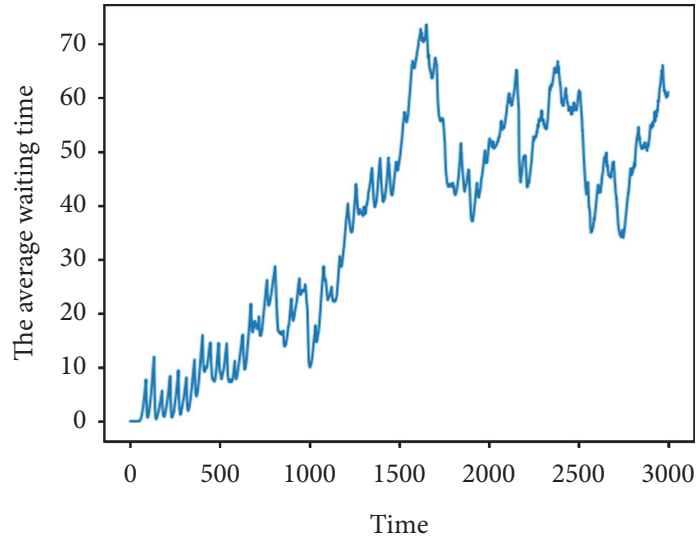

(a)

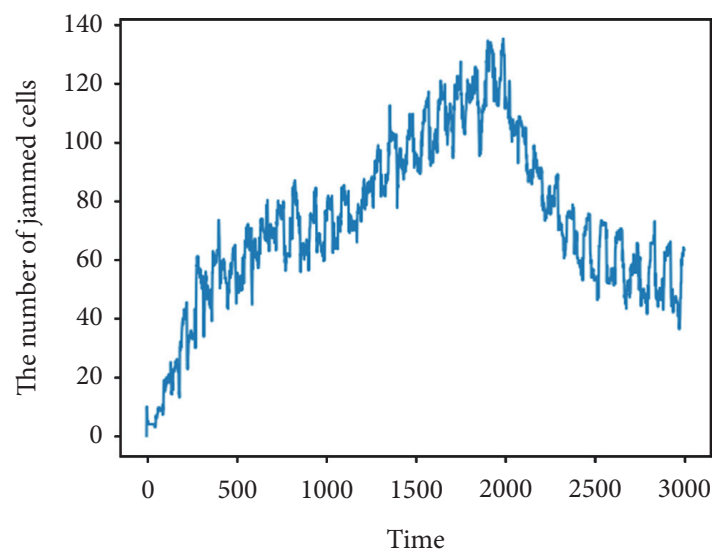

(b)

Figure 13: The number of jammed cells and vehicles' waiting time of a $5 \times 5$ network with fixed time traffic lights. (a) Waiting time. (b) Number of jammed cells.

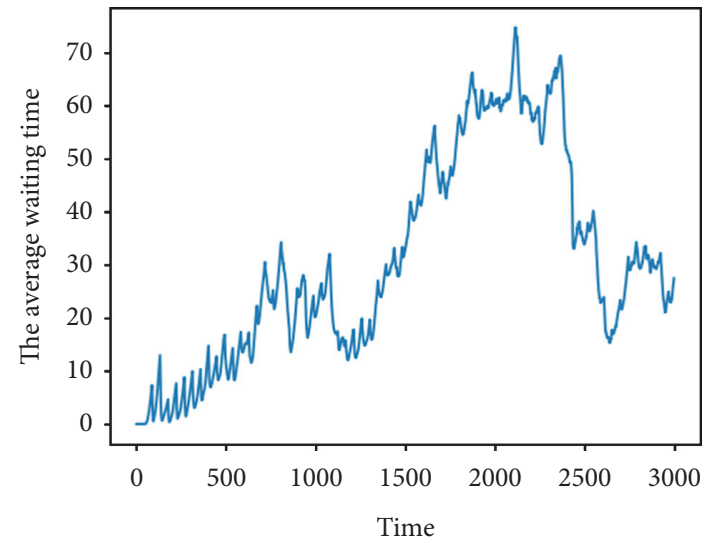

(a)

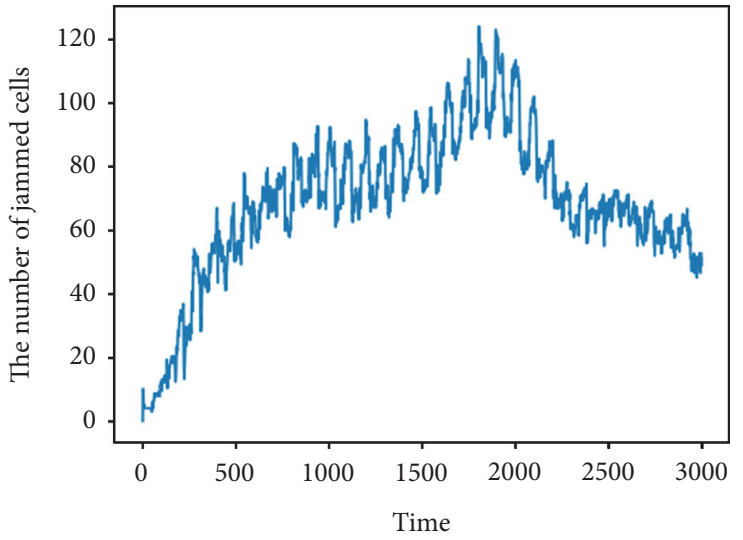

(b)

Figure 14: The number of jammed cells and vehicles' waiting time of a $5 \times 5$ network with ETL strategy and two active ETL in a connected route. (a) Waiting time. (b) Number of jammed cells. 


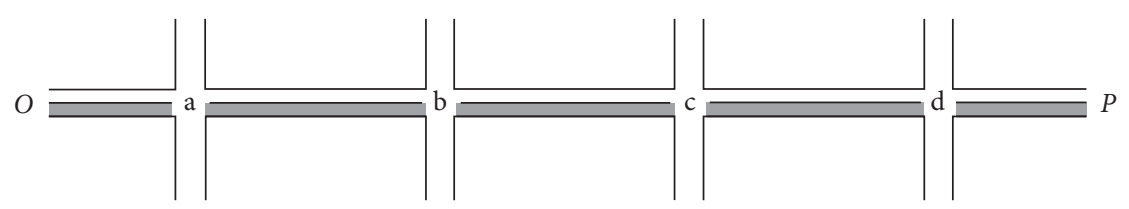

Figure 15: The sample traffic in a subnetwork. The grey links indicate heavy traffic status. In this case, the proposed method gives more green cycle time to vertical routes than horizontal routes. Giving more green cycle time to horizontal routes does not improve traffic status because vehicles cannot move from $(O)$ to $(a)$ and from $(a)$ to $(b)$, while making vertical route green causes to avoid traffic jam on vertical direction.

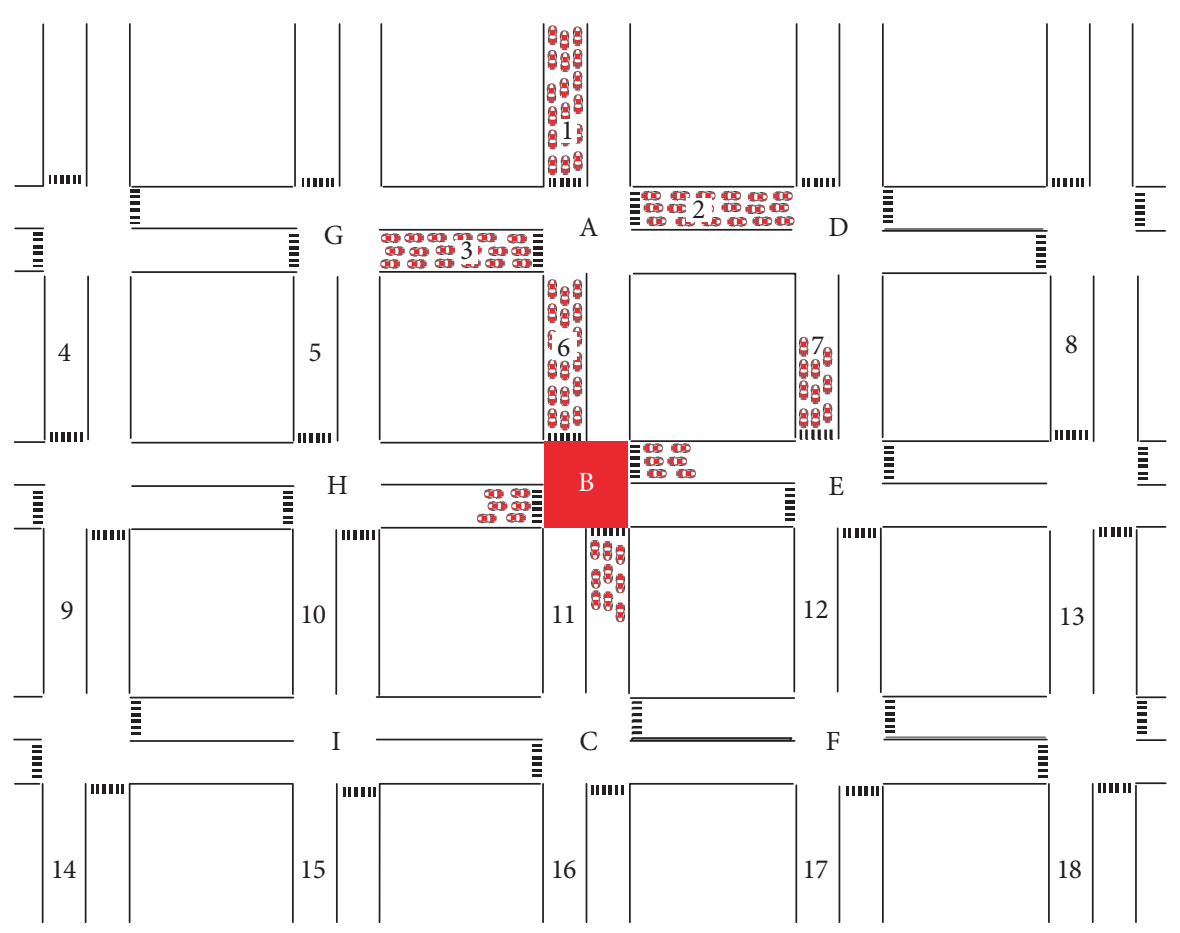

Figure 16: Suppose this network for ETL application. This network has a traffic jam on junction $(B)$. As can be seen, node numbers 1,2 , and 3 are traffic jam providers of traffic jam accrued on junction $(B)$. Using the ELT, the entrance to link number 6 is prevented until the traffic is dissipated. Using this strategy, drivers on links 1,2, and 3 change their route to use alternative paths such as links 5 and 7 . Accordingly, the traffic distributes uniformly in the network to satisfy the user equilibrium principle in the network.

The ETL prevents the drivers to enter a path when the feature links fall in traffic jam. When a junction or a link gets stuck in a traffic jam, the ETL detects which links are directing more traffic flow toward the jammed link or junction. If the number of vehicles that move toward a link exceeds from a predefined threshold $(\theta)$, one or more entrance to that link gets closed using ETL signal. The ETL forces drivers to change their routes and use alternative paths, but the driver whose destination is the ETL-activated link is allowed to enter.

The capacity of each street is $C_{i}=N_{c}^{i} \times \lambda$. Traffic jam is forming on link $i$, if $F C$ ( $i$ ) exceeds $\theta$. For bigger $\theta$, the traffic is probable when FC ( $i$ ) exceeds $\theta$. Hence, the value of $\theta$ in the case of dissipating the traffic jam is lower than the case of avoiding traffic jam. The capacity of each street in our experiment is $9 \times 20=180$. The maximum value of $\theta$ is $180 \times 180=32400$ by considering $l=2$ in (6). However, we can set the model thresholds based on our needs, simply.

\section{Summary and Conclusions}

Traffic light phasing systems are the most available technologies to manage traffic problems in the urban area, all around the world. Traffic light mission is increasing the network capacity and throughput. In this paper, a new traffic light scheduling strategy is proposed, which apparently controls an intersection using local parameters, while handling the global traffic using shared variables with neighbour junctions. In the proposed method, relation matrix is generated based on three parameters, (1) the inflow traffic of a junction, (2) the destination link capacity, and (3) the waiting time on the junction's links. In this model, the inflow traffic of a junction is the destination link capacity of its neighbour junction. This shared parameter causes the model to schedule the traffic light, considering the whole traffic in the network. The proposed traffic light phasing is based on eigenvector centrality measurement. We evaluate 
the method through simulation using SUMO and Python, and the results are compared with two recent literatures. The simulation results approve the efficiency of the proposed method based on various analyses. The ETL is another strategy, which is introduced in this paper. The ETL is a new type of traffic light, which installs at the entrance of a link to show the exit status, with the ability of traffic forecasting. The main goal of ETL is preventing traffic jam by controlling the inflow traffic to a region. To some extent, each traffic light strategy is able to increase the network capacity. The ETL strategy disallows the vehicle entrance into a subnetwork when it predicts a traffic jam in the near future, to hold network highest throughput by preventing additional vehicles entrance and reroute them. However, the ETL is possible to be used beside other traffic light systems to increase the efficiency of a network.

As a future work, we plan to work more on the base model which is presented in (1). In addition, the model is a good candidate to control the green wave, which is included in our future studies. We also expand the idea of ETL in a wider point of views.

As a future work, we will work on ETL long time prediction and survey the effects of changing streets with active ETL to temporal parking.

\section{Data Availability}

No data were used to support this study.

\section{Conflicts of Interest}

The authors declare that there are no conflicts of interest regarding the publication of this paper.

\section{References}

[1] B. L. Ye, W. Wu, K. Ruan et al., "A survey of model predictive control methods for traffic signal control," IEEE/CAA Journal of Automatica Sinica, vol. 6, no. 3, 2019.

[2] D. I. Robertson and R. D. Bretherton, "Optimizing networks of traffic signals in real time-the scoot method," IEEE Transactions on Vehicular Technology, vol. 40, no. 1, pp. 11-15, 1991.

[3] P. R. Lowrie, SCATS: A Traffic Responsive Method of Controlling Urban Traffic Control, New South Wales, Roads and Traffic Authority, Canberra, Australia, 1992.

[4] S.-F. Cheng, M. A. Epelman, and R. L. Smith, "CoSIGN: a parallel algorithm for coordinated traffic signal control," IEEE Transactions on Intelligent Transportation Systems, vol. 7, no. 4, pp. 551-564, 2006.

[5] K. Gao, Y. Zhang, A. Sadollah, and R. Su, "Optimizing urban traffic light scheduling problem using harmony search with ensemble of local search," Applied Soft Computing, vol. 48, pp. 359-372, 2016.

[6] G. Improta and G. E. Cantarella, "Control systems design for an individual signalized junction," Transportation Research Part B: Methodological, vol. 18, pp. 147-167, 1982.

[7] M.-T. Li and A. C. Gan, "Signal timing optimization for oversaturated networks using TRANSYT-7F," Transportation Research Record: Journal of the Transportation Research Board, vol. 1683, no. 1, pp. 118-126, 1999.
[8] D. I. Robertson, "Transyt method for area traffic control," Transport Research International Documentation, vol. 11, no. 6, pp. 276-281, 1996.

[9] P. B. Hunt, D. L. Robertson, and R. D. Bretherton, "The scoot on-line traffic signal optimization technique," Traffic Engineering and Control, vol. 23, pp. 190-192, 1982.

[10] N. H. Gartner, "OPAC: a demand-responsive strategy for traffic signal control," Transportation Research Record Journal of the Transportation Research Board, vol. 906, 1983.

[11] J. L. Farges, J. J. Henry, and J. Tufal, "The PRODYN real-time traffic algorithm," in Proceedings of the 4th IFAC Symposium, pp. 307-312, Stuttgart, Germany, April 1983.

[12] F. Boillot, J. M. Blosseville, J. B. Lesort, V. Motyka, M. Papageorgiou, and S. Sellam, "Optimal signal control of urban traffic networks," in Proceeding of the 6th IEEE Intenational Conference Road Traffic Monitoring and Control, pp. 75-79, Cracow, Poland, 1992.

[13] S. Sen and K. L. Head, "Controlled optimization of phases at an intersection," Transportation Science, vol. 31, no. 1, pp. 5-17, 1997.

[14] K. Gao, Y. Zhang, R. Su, F. Yang, P. N. Suganthan, and M. Zhou, "Solving traffic signal scheduling problems in heterogeneous traffic network by using meta-heuristics," IEEE Transactions on Intelligent Transportation Systems, vol. 20, no. 9, pp. 1-11, 2018.

[15] J. J. Shachez, M. Gal, and E. Rubio, "Genetic algorithms and cellular automata: a new architecture for traffic light cycles optimization," in Proceedings of the 2004 Congress on Evolutionary Computation, pp. 1668-1674, Portland, OR, USA, June 2004.

[16] J. Garca Nieto, A. Carolina Olivera, and E. Alba, "Optimal cycle program of traffic lights with particle swarm optimization," IEEE transaction on Evolutionary Computation, vol. 17, no. 6, pp. 823-839, 2013.

[17] Y. S. Huang, Y. S. Weng, and M. Zhou, "Design of regulatory traffic light control systems with synchronized timed petri nets," Asian Journal of Control, vol. 20, no. 2, pp. 1-12, 2018.

[18] L. Qi, M. Zhou, and W. Luan, "Impact of driving behavior on traffic delay at a congested signalized intersectionfic delay at a congested signalized intersection," IEEE Transactions on Intelligent Transportation Systems, vol. 18, no. 7, pp. 1882-1893, 2017.

[19] A. Stevanovic, Adaptive Traffic Control Systems: Domestic and Foreign State of Practice, Transportation Research Board, Washington, DC, USA, 2010.

[20] S. Choi, B. B. Park, J. Lee, H. Lee, and S. H. Son, "Field implementation feasibility study of cumulative travel-time responsive (CTR) traffic signal control algorithm," Journal of Advanced Transportation, vol. 50, no. 8, pp. 2226-2238, 2016.

[21] F. C. Fang and L. Elefteriadou, "Modeling and simulation of vehicle projection arrival-discharge process in adaptive traffic signal controls," Journal of Advanced Transportation, vol. 44, no. 3, pp. 176-192, 2010.

[22] W. Huang, F. Viti, and C. M. J. Tampère, "A dual control approach for repeated anticipatory traffic control with estimation of network flow sensitivity," Journal of Advanced Transportation, vol. 50, no. 7, pp. 1386-1412, 2016.

[23] K. Aboudolas, M. Papageorgiou, and E. Kosmatopoulos, "Store-and-forward based methods for the signal control problem in large-scale congested urban road networks," Transportation Research Part C: Emerging Technologies, vol. 17, no. 2, pp. 163-174, 2009.

[24] D. I. Robertson, "TRANSYT: a traffic network study tool," Technical Report, Transportation Research Board, Washington, DC, USA, 1969. 
[25] N. H. Gartner and C. Stamatiadis, "Arterial-based control of traffic flow in urban grid networks," Mathematical and Computer Modelling, vol. 35, no. 5-6, pp. 657-671, 2002.

[26] I. Kosonen, "Multi-agent fuzzy signal control based on realtime simulation," Transportation Research Part C: Emerging Technologies, vol. 11, no. 5, pp. 389-403, 2003.

[27] V. C. Pham, F. Alam, J. Potgieter, F. C. Fang, and W. L. Xu, "Integrated fuzzy signal and ramp-metering at a diamond interchange," Journal of Advanced Transportation, vol. 47, no. 4, pp. 413-434, 2013.

[28] W. Yang, L. Zhang, and Z. He, "Rolling-horizon optimization based three-stage fuzzy logic controller for urban traffic signals," Journal of Tongji University Natural Science, vol. 42, no. 12, pp. 1846-1853, 2014.

[29] R. Armas, H. Aguirre, F. Daolio, and K. Tanaka, "Evolutionary design optimization of traffic signals applied to Quito city," PLoS One, vol. 12, no. 12, 2017.

[30] S. Lawe and R. Wang, "Optimization of traffic signals using deep learning neural networks," in Proceedings of the Australasian Joint Conference on Artificial Intelligence, pp. 403415, Adelaide, Australia, 2016.

[31] L. Li, Y. Lv, and F. W. Wang, "Traffic signal timing via deep reinforcement learning," IEEE/CAA Journal of Automatica Sinica, vol. 3, no. 3, pp. 247-254, 2016.

[32] J. Zhao, Y. Liu, and P. Li, "A network enhancement model with integrated lane reorganization and traffic control strategies," Journal of Advanced Transportation, vol. 50, no. 6, pp. 1090-1110, 2016.

[33] Z. Li and P. Schonfeld, "Hybrid simulated annealing and genetic algorithm for optimizing arterial signal timings under oversaturated traffic conditions," Journal of Advanced Transportation, vol. 49, no. 1, pp. 153-170, 2015.

[34] J. C. Spall and D. C. Chin, "Traffic-responsive signal timing for system-wide traffic control," Transportation Research Part C: Emerging Technologies, vol. 5, no. 3, pp. 153-163, 1997.

[35] S. P. Hoogendoorn, V. L. Knoop, and H. J. Van Zuylen, "Robust control of traffic networks under uncertain conditions," Journal of Advanced Transportation, vol. 42, no. 3, pp. 357-377, 2008.

[36] D. Srinivasan, M. C. Choy, and R. L. Cheu, "Neural networks for real-time traffic signal control," IEEE Transactions on Intelligent Transportation Systems, vol. 7, no. 3, pp. 261-272, 2006.

[37] E. B. Kosmatopoulos, M. M. Polycarpou, M. A. Christodoulou, and P. A. Ioannou, "High-order neural network structures for identification of dynamical systems," IEEE Transactions on Neural Networks, vol. 6, no. 2, pp. 422-431, 1995.

[38] C. F. A. Negre, U. N. Morzan, H. P. Hendrickson et al., "Eigenvector centrality for characterization of protein allosteric pathways," Proceedings of The National Academy of Sciences, vol. 115, no. 52, pp. E12201-E12208, 2018.

[39] M. E. Newman, The Mathematics of Networks, Springer, Berlin, Germany, 2015.

[40] J. Long, Z. Gao, X. Zhao, A. Lian, and P. Orenstein, "Urban traffic jam simulation based on the cell transmission model," Networks and Spatial Economics, vol. 11, no. 1, pp. 43-64, 2011.

[41] C. F. Daganzo, "The cell transmission model: a dynamic representation of highway traffic consistent with the hydrodynamic theory," Transportation Research Part B: Methodological, vol. 28, no. 4, pp. 269-287, 1994.

[42] C. F. Daganzo, "The cell transmission model, part II: network traffic," Transportation Research Part B: Methodological, vol. 29, no. 2, pp. 79-93, 1995.
[43] M. J. Lighthill and G. B. Whitham, "On kinematic waves. II: a theory of traffic flow on long crowded roads," Proceedings of The Royal Society A Mathematical Physical and Engineering, vol. 229, pp. 317-345, 1955.

[44] L. Qi, M. Zhou, and W. Luan, "A two-level traffic light control strategy for preventing incident-based urban traffic congestion," IEEE Transaction on Intelligent Transportation Systems, vol. 19, no. 1, 2018.

[45] F. Yan, G. Yan, M. Ren, J. Tian, and Z. Shi, "A novel control strategy for balancing traffic flow in urban traffic network based on iterative learning control," Physica A: Statistical Mechanics and Its Applications, vol. 508, pp. 519-531, 2018.

[46] J. Haddad and N. Geroliminis, "On the stability of traffic perimeter control in two-region urban cities," Transportation Research Part B: Methodological, vol. 46, no. 9, pp. 1159-1176, 2012. 